\title{
El contrato de confianza con la información televisiva
}

\author{
Javier CALlejo GaLlego \\ Universidad Nacional de Educación a Distancia (UNED) \\ mcallejo@poli.uned.es
}

Recibido: 4 de abril de 2014

Aceptado: 30 de octubre de 2014

\begin{abstract}
Resumen
El trabajo aborda la confianza que la sociedad española deposita en las noticias televisivas. Desde la relativa fidelidad a los distintos programas informativos de las respectivas cadenas televisivas, cabe establecer la hipótesis del dominio de la confianza en la cadena sobre sus elementos (programas, profesionales). Partiendo de las relativas limitaciones del enfoque luhmaniano para dar cuenta de la confianza con respecto al sistema de comunicación mediada organizada, se realiza una aproximación empírica a la percepción que tiene el público de las noticias televisivas. Las fuentes empíricas principales son diversas encuestas del Centro de Investigaciones Sociológicas y seis grupos de discusión entre sectores de la clase media, con distintas edades, llevados a cabo en Madrid, y centrados en la información en televisión.
\end{abstract}

Palabras clave: Información televisiva, públicos, confianza, sistema de comunicación.

\section{The contract of trust with television news}

\begin{abstract}
The work focuses on the trust that society places on the Spanish television news. From the relative fidelity to the various news programs of the respective television networks, it can be supposed the domain of the trust in television networks above the trust in its programmes and professionals. Based on the relative limitations of luhmanian approach to account for the trust with regard to organized mediated communication system, it is made an empirical approach to the perception of the public television news. The main empirical sources are various surveys of Centro de Investigaciones Sociológicas and six focus groups among the middle class, with different ages. They were held in Madrid and focused on information in television.
\end{abstract}

Keywords: Television news, publics, trust, communication system.

\section{Referencia normalizada}

CALLEJO GALLEGO, Javier (2015): "El contrato de confianza con la información televisiva". Estudios sobre el Mensaje Periodístico. Vol. 21, Núm. 2 (julio-diciembre), págs.: 969-986. Madrid, Servicio de Publicaciones de la Universidad Complutense.

Sumario: 1. Introducción: el significado de la confianza en las noticias. 2. Planteamiento y metodología de la investigación. 3. Noticias de verdad como núcleo del informativo. 4. La verdad de las noticias: elementos para la confianza en las noticias. 5. Conclusiones. 6. Referencias bibliográficas.

\section{Introducción: el significado de la confianza en las noticias}

¿Qué significa la confianza en un medio de comunicación? La principal manifestación de la confianza en un medio de comunicación está en la selección del mismo, en un contexto de pluralidad y de competencia entre medios de comunicación. Razón por la que no haría falta preguntar a los públicos por la confianza. Sin embargo, una exigencia de mayor precisión conceptual conduce a desistir de la identificación entre seguimiento y confianza. Ni siquiera entre dos conceptos tan próximos como fidelidad a un medio y confianza. Cabe esperar cierto solapamiento entre estos conceptos, pero 
no son lo mismo, como no se podría entender que la confianza en el Boletín Oficial del Estado conduzca a un seguimiento fiel de este medio.

La confianza, especialmente en el campo de la noticia, parece venir dada inicialmente en clave de verdad. Es confianza en la verdad de la noticia. Y ello porque, como dice Luhmann (2000), solo nos enteramos de lo que pasa en la sociedad a través de los medios de comunicación. Es la relación con lo que pasa lo que parece constituir la base de la confianza. Una relación que dista de ser evidente debido a que incluye la relación de lo que pasa -qué es lo que se selecciona como información entre todo lo que ocurre en la realidad social-y la relación entre el mensaje sobre lo que se selecciona como ocurrido y lo ocurrido.

¿Es importante la confianza en el análisis de los medios de comunicación? Lo es para el funcionamiento de los medios de comunicación, ya sean tomados como conjunto, formando un sistema social -aquí adquiere la denominación de sistema de comunicación mediada organizada, ya sean tomados individualmente. Natalie Nourgayréde, directora de Le Monde, manifestaba en una entrevista ${ }^{1}$ que la estrategia de su medio residía en la constitución de un contrato de confianza.

La confianza está vinculada con la incertidumbre (Luhmann, 1996; Stozmpka, 1999), aún cuando las teorías discutan las reglas de tal vinculación. Si la incertidumbre de los públicos puede estar en el grado de verdad de lo que cuentan los medios de comunicación, éstos tienen la incertidumbre de que sus productos -en este caso las noticias- sean recibidos por el público. Incertidumbres que parecen tener su relativa articulación pragmática: los públicos seguirán las noticias de los medios en la medida que confían en ellos, y que, por lo tanto, se sientan perceptores de verdad; y, a su vez, los medios podrían confiar en la existencia de un número más o menos estable ${ }^{2}$ de seguidores de sus noticias durante relativos períodos de tiempo. Pues bien, hay que destacar que la relación más estable de un medio de comunicación con la sociedad, y, por lo tanto, con el público, tiende a encontrarse en los programas informativos, como muestra el hecho de que, a pesar de la gran multiplicación de oferta, sigan estando entre los programas más seguidos por la audiencia ${ }^{3}$. Por lo tanto, la confianza para los medios es fuente de estabilidad y de cierta reducción del sentimiento de incertidumbre en sí mismo, ya que no de la incertidumbre en sí misma, ya que la confianza es una forma de seguridad (Luhmann, 1996: 20). En todo caso, como resalta Thuderoz (2003: 19), la confianza es una categoría importante de nuestras prácticas.

Debido a su relevancia pragmática, la confianza en la noticia ha sido aborda por autores tan prestigiosos como Schudson (1978). A la vez, al tratarse de un estado variable en la relación entre medios de comunicación y sociedad, conviene actualizar tal

1 El País, 7 de abril de 2013.

2 La estabilidad se deriva también de la rutina, de la estructuras temporales; pero esto explicaría más el hecho de que las cadenas de televisión coincidan en los momentos del día que asignan para la emisión de estos programas informativos, y no el hecho de que se seleccionen unos programas informativos en lugar de otros.

3 Al menos suele haber tres informativos diarios entre los diez programas más vistos durante el día en el conjunto de la televisión española, según los datos de medición de audiencia mediante audímetro de la empresa Kantar Media. Última comprobación: 28 de marzo de 2014. 
estado en nuestro país. Al fin y al cabo, siguiendo al propio Luhmann (1996), toda confianza es siempre en el presente, aunque su situación se pueda explicar por procesos que tienen su raíz en el pasado (Stozmpka, 1999: 127 y ss.). La confianza en los medios de comunicación no ha de ser considerada muy distinta de la confianza en la ciencia, y ésta ha cambiado a lo largo del tiempo (Ziman, 2000).

Resulta importante destacar cómo históricamente todos los medios de comunicación han nacido bajo la desconfianza. Todos los medios de comunicación han nacido enfrentados a la desconfianza y con lo que Livingstone (2002) denomina pánico moral, en cuanto la preocupación por proteger a jóvenes y niños de la acción de los medios de comunicación recién nacidos tiende a prevalecer. Hasta la escritura, el texto escrito, tuvo que vencer a la desconfianza con que fue recibido (Lledó, 1998). Sucesivamente ocurrió con los diarios, la radio, el cine, la televisión y, hoy, con internet (Briggs y Burke, 2002). Tal vez debido, como señala Maigret (2005: 36), a una desconfianza originada reactivamente por las instituciones escolares, tendentes a ser fuente de la legitimidad de las prácticas culturales (Bourdieu, 1988; Lahire, 2006).

El primer signo para la fiabilidad que ofrece el sistema de comunicación mediada de producción y distribución de noticias, es su propia periodicidad, en el siglo XVIII (Frank, 1961; Mott, 1962). No faltan bromas con respecto a esto, como quienes señalaban que la única verdad de un diario era el nombre del mismo y la fecha. Pero una vez que la periodicidad se ha institucionalizado, hasta poder ser considerada el núcleo del sistema de comunicación mediada organizada, queda por observar cuáles son las otras fuentes de confianza.

En este trabajo, el objeto sobre el que se cuestiona la confianza no son tanto los medios de comunicación, como la noticia en un medio específico. Hay que reconocer que no es un objeto cualquiera dentro del sistema de comunicación mediada, sino que es su producto vertebral (McQuail, 1991: 2; Benito, 1995: 90), lo que hace que, a su vez, la observación de la confianza o desconfianza que generan las noticias se convierta en observación de la confianza o desconfianza sobre el conjunto de medios de comunicación.

La noticia como objeto de confianza de la sociedad ha pivotado principalmente sobre la selección de las mismas, más que sobre su verdad. Cabe recordar aquí el trabajo etnográfico de Tuchman (1983) sobre los procesos de selección de las noticias en las redacciones periodísticas, en cuanto puede considerarse que subyace el cuestionamiento de tal selección. Lo que se cuestiona es si lo que se selecciona de la realidad es lo importante. Si no es lo importante, se abre el margen para las sospechas y la desconfianza, como desarrollan Galtung y Ruge (1965) con respecto a las páginas de información internacional de los periódicos. La fuente de valor de la noticia está en su importancia (Wolf, 1987: 223), por lo que es coherente plantear que también está ahí la base para su confianza. Ahora bien ¿qué es lo importante? ¿Todos los actores se refieren a lo mismo cuando señalan lo importante? Hace medio siglo, Clausse (1961) subraya el gap entra las teorías de la noticia (entre el consejo sociológico y humanista de la noticia), la investigación empírica sobre lo que los periodistas entienden y practican como noticia y las investigaciones de mercado (literatura gris) sobre las preferencias de la audiencia de noticias. Es en este último acto en que se centra este trabajo ¿Qué es lo que consideran los públicos como importante y, por lo 
tanto, confiable? Las múltiples denuncias, casi siempre desde una perspectiva normativa y profesional, a la tendencia de la información, especialmente en el medio televisivo (Blumler, 1993; McManus, 1994; Franklin, 1997; Langer, 1998; Brants, 1998; Winston, 2002; Thussu, 2007), parecen marcar una distancia entre lo que debiera considerarse importante y lo que se proyecta que los públicos consideran importante y, por lo tanto, noticiable y, de esta manera, digno de confianza.

¿Por qué la confianza? Hasta finales de los años setenta, la confianza ha ocupado un lugar secundario en las preocupaciones sociológicas y, cuando lo hacía, tendía a actuar más cerca de la psicología social -con especial foco en las situaciones microsociológicas- que como sociología de los procesos sociales. Es la obra de Luhmann (1992; 1996), la que rompe con tal situación, siendo, además, interpretada (Sztompka, 1999) como una aproximación de la sociología a las preocupaciones más generales y cotidianas de la sociedad. A partir de entonces, la observación de la confianza queda integrada en la mayor parte de las escuelas y corrientes sociológicas y, de alguna manera, queda atravesada por sus divisiones. En el intento de articular tales divisiones en torno a la confianza, el polaco Piotr Sztompka señala una doble cara de la confianza según se encuentre vinculada a (y explicada desde) la racionalidad de la acción, que es la línea reinaugurada por el funcionalismo del propio Luhmann, o que demande la búsqueda del sentido que los sujetos dan a las prácticas de la confianza, desde una cultura de la confianza, que se vincula a los estudios que relacionan cultura de la confianza en una sociedad con salud democrática (Almond y Verba, 1989; Putnam, 1995 y 2000; Inglehart, 1999). De una manera un tanto esquemática, puede decirse que desde la primera cara se pregunta por qué debemos de confiar y, por lo tanto, qué funciones cumple la confianza; mientras que por el segundo la cuestión es por qué los sujetos confian en lo que dicen confiar. Siguiendo la estela del propio Szotmpka (1999), lo que aquí se intenta es la articulación de esas dos perspectivas de la confianza.

Si la confianza en la noticia está vinculada a la verdad de la noticia ¿Qué significa la verdad de la noticia? Desde su dimensión referencial, lo que se cuenta en esos mensajes denominados noticias es verdad y, por lo tanto, referencia o describe una realidad. Ahora bien, reducir el papel de los medios de comunicación a contar la verdad es, como poco, exagerado, y, en todo caso, apenas diferenciaría la lógica social de los medios de comunicación de la de otras instituciones sociales. Es aquí donde convenga analizar el contenido de esa verdad de la noticia más allá de su carácter referencial. Por un lado, la verdad de la noticia desde la perspectiva de la selección de una realidad como noticia. Desde aquí, la verdad de la noticia es una noticia de verdad, de que lo que se cuenta es realmente reseñable y digno, por su relevancia, de formar parte del discurso informativo de los medios de comunicación. Una verdad de la noticia que tiene que ver con la función de vigilar el entorno (Lasswell, 1982) y ordenar la realidad, el entorno social, atribuida a los medios de comunicación, por la que los receptores confían en que lo que los medios de comunicación seleccionan de la realidad como noticia es una noticia. De que se trata de una noticia de verdad. Ahora bien, desde la concepción de los múltiples sentidos que pueden imprimirse a una misma realidad seleccionada como noticia, la verdad de la noticia puede entenderse como la fijación del sentido a esa realidad, un sentido producido -al menos, inicialmente- por 
el emisor. ¿Hasta qué punto la proyección de confianza en los medios de comunicación está dirigida hacia un significado de la verdad de la noticia o hacia otros significados? ¿Cuáles son las fuentes de confianza -y desconfianza- en uno y otro significado de la verdad?

Por lo tanto y como resumen de lo anterior, abordar la confianza que tiene la sociedad en las noticias ofrecidas por un medio como la televisión es, en primer lugar, plantear la relación de la sociedad con el conjunto del sistema de comunicación mediada organizada y, por lo tanto, con los medios en general. En segundo lugar, plantear la relación con un medio específico, como es la televisión. Un paso que deriva del anterior. Asimismo, como toda confianza, conlleva representaciones del objeto -la noticia televisiva - y pone en juego la confianza en los sujetos implicados en la relación, como son los profesionales cuando se trata de un sistema experto como el de los medios de comunicación. Es decir, la confianza en los periodistas. Por último, entrar en la confianza que los distintos públicos tienen en las noticias televisivas es abrir la posibilidad de observar cuáles son los elementos del mensaje periodístico televisivo que generan respectivamente confianza y desconfianza. Más allá de la concreción del objeto que aquí nos ocupa, toda aproximación a la concreción de la confianza señala las reglas que rigen la cultura de la confianza (Stozmpka, 1999: 101) y, por lo tanto, el estado de tal cultura en una determinada sociedad en un específico momento de su historia.

\section{Planteamiento y metodología de la investigación}

Según los resultados de las encuestas del Centro de Investigaciones Sociológicas (CIS), la confianza en los medios de comunicación está en el fiel de la balanza. Prácticamente la mitad $(49,8 \%)$ se sitúa en los valores medios de la escala $(4,5$ y 6$)$, el $24,3 \%$ se sitúa por debajo de esos valores y por lo tanto proyectan una muy escasa confianza en los medios de comunicación; y un 22,6\%, por encima, mostrando bastante confianza (Tabla 1). Si nos fijamos en la evolución de la media, se percibe su escasa variabilidad (gráfico 1), entre el 4,31 (entre 0 y 10) de diciembre de 2009 y el 5,20 de octubre de 2007. En los resultados más recientes, de abril de 2013, la media es de 4,79 , que se sitúa prácticamente en el punto medio de esos valores "extremos".

Tabla 1: Grado de confianza (entre 0 y 10) en los medios de comunicación (porcentajes en vertical, media y desviación típica)

\begin{tabular}{|c|r|r|r|r|r|r|r|r|r|r|r|}
\hline Mes-año & ene-05 & ene-06 & oct-06 & may-07 & oct-07 & feb-09 & dic-09 & nov-10 & oct-11 & nov-11 & abr-13 \\
\hline $\mathbf{N}^{\mathbf{0}}$. Estudio & 2588 & 2632 & 2657 & 2701 & 2736 & 2790 & 2826 & 2853 & 2914 & 2920 & 2984 \\
\hline $\mathbf{( 0 - 1 )}$ & 10,6 & 4 & 9,9 & 10,6 & 7,4 & 9,3 & 13,8 & 13,6 & 10,8 & 11,9 & 11,3 \\
\hline $\mathbf{( 2 - 3 )}$ & 11,9 & 10,1 & 12,3 & 11,5 & 10,9 & 11,9 & 16,5 & 14,5 & 12,4 & 16,2 & 13,0 \\
\hline $\mathbf{( 4 - 5 - 6 )}$ & 47,3 & 50,6 & 52,9 & 48,7 & 51,4 & 52,2 & 50,2 & 47,2 & 47,7 & 50,6 & 49,8 \\
\hline $\mathbf{( 7 - 8 )}$ & 18,2 & 24,6 & 18,1 & 17,4 & 19,9 & 18,1 & 12,6 & 16,5 & 19,9 & 14,4 & 18,5 \\
\hline $\mathbf{( 9 - 1 0 )}$ & 3,5 & 5,4 & 3,2 & 5,5 & 5,3 & 3,5 & 2,2 & 4,1 & 5,7 & 3,0 & 4,1 \\
\hline N.S. & 7,3 & 4,2 & 2,9 & 5,5 & 4,2 & 4,1 & 4,2 & 3,6 & 2,9 & 3,9 & 3,0 \\
\hline N.C. & 1,2 & 1 & 0,7 & 0,8 & 0,7 & 0,9 & 0,5 & 0,6 & 0,6 & 0,1 & 0,2 \\
\hline (N) & 2479 & 3192 & 2480 & 2983 & 3716 & 2978 & 2478 & 2469 & 2472 & 6082 & 2482 \\
\hline Media & 4,82 & 5,54 & 4,84 & 4,92 & 5,2 & 5,03 & 4,31 & 4,58 & 4,97 & 4,52 & 4,79 \\
\hline Desv. Tip. & 2,36 & 2,12 & 2,29 & 2,44 & 2,26 & 2,22 & 2,33 & 2,49 & 2,47 & 2,31 & 2,38 \\
\hline $\mathbf{N}$ & 2269 & 3025 & 2391 & 2795 & 3532 & 2829 & 2360 & 2365 & 2385 & 5838 & 2401 \\
\hline
\end{tabular}

Fuente: Elaboración propia a partir del banco de datos del Centro de Investigaciones Sociológicas 


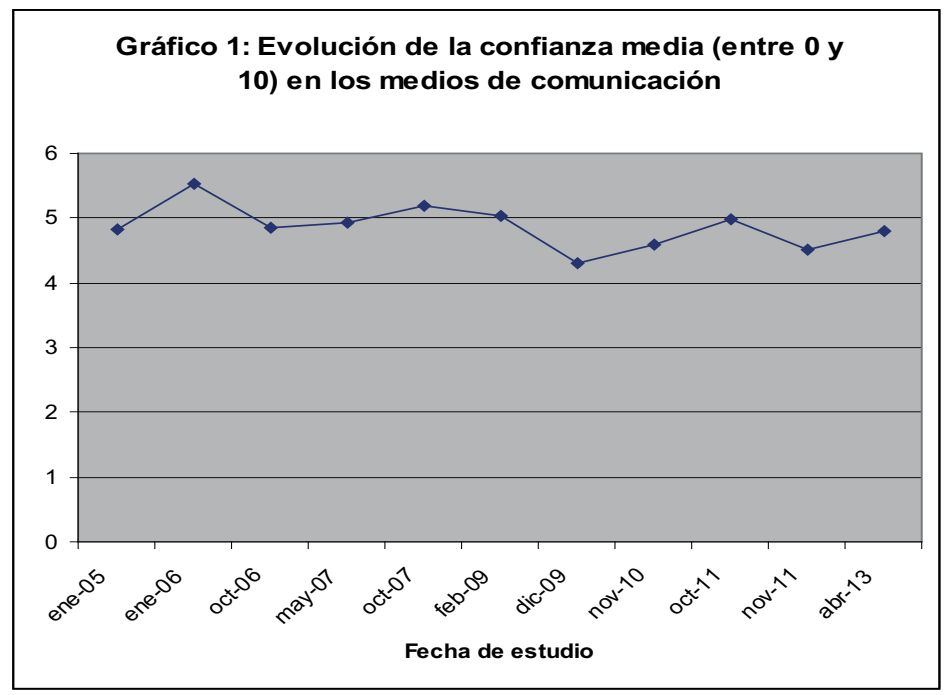

En principio, podría hablarse de una baja confianza o de una confianza en estado crítico con respecto a los medios de comunicación; pero bajo tal estabilidad relativa se muestra una posición de la sociedad con respecto a los medios. Una estabilidad que hay que comparar con las fluctuaciones de la confianza en otras instituciones, especialmente las relacionadas con la política (Parlamento, partidos políticos, sindicatos, Monarquía, etc.), y con el propio grado de confianza con respecto a estas instituciones en los registros más recientes. De hecho, la confianza en los medios de comunicación se sitúa solo detrás de la proyectada en la Guardia Civil $(5,71)$, la Policía $(5,65)$ y las Fuerzas Armadas $(5,21)$ en la encuesta de abril de 2013. Bastante por encima de la confianza en instituciones como el Parlamento $(2,53)$, el Defensor del Pueblo $(3,94)$ o los partidos políticos $(1,83)$.

La generalización de la categoría medios de comunicación puede esconder una muy distinta confianza a los diversos tipos de medios de comunicación y, por su puesto, con respecto a un medio de comunicación en particular. Aquél que se sigue habitualmente es difícil que pueda ser incluido por los entrevistados entre los medios que se consideran con baja confianza, puesto que, en tal caso, no se seguiría. En cuanto al tipo de medios de comunicación, la radio adquiere mayor confianza que los diarios a la hora de obtener confianza por los ciudadanos. Así, en el año 2010 (estudio n ${ }^{\circ}$. 2849, Latinobarómetro, último resultado presente con esta referencia en el banco de datos del CIS), el porcentaje de quienes muestran mucha o alguna confianza hacia la radio asciende al 54,3\%; mientras que desciende al $43,2 \%$ para los periódicos y al $36,4 \%$ para el caso de la televisión. En los tres medios de comunicación, por encima de la confianza mostrada hacia el Gobierno ( $25,6 \%$ manifiestan mucha o alguna confianza) o los bancos $(21,3 \%)$, y por debajo de la proyectada hacia la policía $(71,2 \%)$.

Centrados en la televisión, puede observarse (Tabla 2, gráfico 2) cómo parece haber generado más desconfianza que confianza, encontrándose los que confian mucho o 
algo en la televisión por debajo del $50 \%$ en todos los estudios registrados por el CIS desde 1997. No obstante, sus peores registros se encuentran en los estudios del año 2003. Lo que hay que vincular a un descenso general en la denominada cultura de la confianza (Stzompka, 1999) del país.

Tabla 2: Grado de confianza (mucha o alguna y poca o ninguna) en la televisión (porcentajes en vertical)

\begin{tabular}{|l|r|r|r|r|r|r|r|r|r|r|r|r|}
\hline \multicolumn{1}{|c|}{ mes-año } & \multicolumn{1}{c|}{ jul-96 } & dic-1997 & may-01 & ene-03 & oct-03 & sep-04 & oct-05 & nov-06 & nov-07 & dic-08 & nov-09 & oct-10 \\
\hline $\mathrm{N}^{\text {o. Estudio }}$ & 2218 & 2270 & 2417 & 2476 & 2540 & 2571 & 2620 & 2663 & 2741 & 2777 & 2823 & 2849 \\
\hline Mucha/alguna & 44,6 & 41,4 & 49,5 & 35,5 & 32,0 & 27,2 & 38,7 & 46,4 & 38,0 & 43,8 & 38,9 & 36,4 \\
\hline Poca/ninguna & 51,5 & 55,1 & 48,2 & 62,8 & 65,8 & 68,2 & 59,6 & 51,6 & 59,6 & 53,3 & 59,2 & 61,9 \\
\hline (N) & 2481 & 2476 & 2496 & 2484 & 2495 & 2491 & 3033 & 2476 & 2482 & 2471 & 2486 & 2483 \\
\hline
\end{tabular}

Fuente: Elaboración propia a partir del banco de datos del Centro de Investigaciones Sociológicas

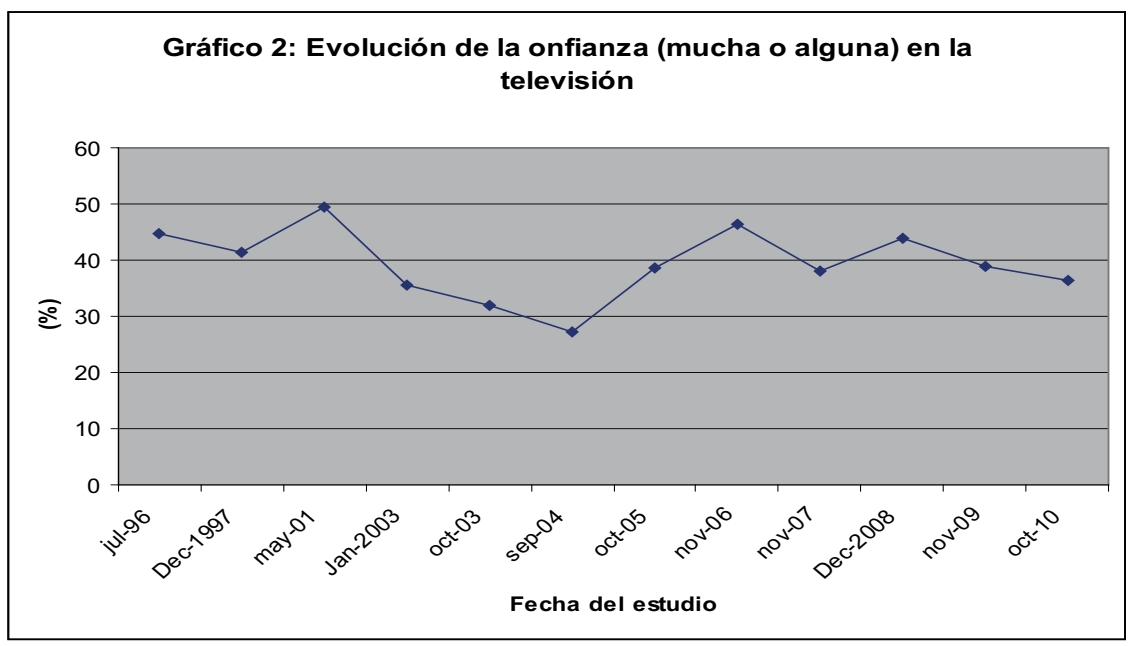

Por lo tanto, tenemos que, según estos estudios a través de encuesta con cuestionario estandarizado, la confianza en los medios de comunicación puede considerarse como una confianza crítica (Simmel, 2013; Löfstedt, 2005): hay confianza y aunque es superior a la que se muestra con respecto a otras instituciones se encuentra en unos umbrales mínimos, existiendo prácticamente la misma proporción entre quienes confían y entre quienes desconfían. Una desconfianza que es más patente en el caso de la televisión. Partiendo del carácter vertebral que tiene la información en la construcción de la representación social de los medios y, por lo tanto, de la confianza hacia los mismos y de la relación con ellos, se deriva la pregunta central de la investigación: cuál es la confianza hacia las noticias televisivas y la base de la misma. Pregunta de investigación que se ha trasladado a la perspectiva cualitativa de investigación social.

Los resultados que se analizan a partir de aquí proceden de un conjunto de seis grupos de discusión, llevados a cabo durante el año 2009, pertenecientes a un estudio sobre la percepción de la evolución de los programas informativos en televisión ${ }^{4}$ en

${ }^{4}$ El estudio fue realizado por un equipo dirigido por el profesor Luis Pablo Francescutti, de la Universidad Rey Juan Carlos de Madrid, y del que el autor de este trabajo formó parte. 
España. Como ocurre generalmente en los estudios cualitativos de audiencia, se ha trabajo con lo que cabe asumir como sectores centrales de la sociedad. Puede decirse que son grupos típicos que representan posiciones más o menos directamente determinables en la fracción media de la estructura social, de manera que cabe suponer que los participantes en las reuniones grupales comparten discursos sobre el objeto de investigación, en función de que comparten posición en la estructura social. Por lo tanto, los grupos son representativos de la clase media en sentido amplio y fueron realizados en Madrid, tomando como criterios para su diseño: sexo, edad, ocupación e informativo televisivo habitualmente seguido, conformándose las siguientes reuniones de grupo:

\begin{tabular}{|c|c|}
\hline $\begin{array}{l}\text { GRUPOS DE } \\
\text { DISCUSIÓN }\end{array}$ & CARACTERÍSTICAS \\
\hline $\begin{array}{ll}\text { Grupo } & \text { de } \\
\text { Discusión } 1 & \end{array}$ & $\begin{array}{l}\text { Grupo de hombres y mujeres, ocupados, entre } 30 \text { y } 40 \text { años, trabajen como técnicos o } \\
\text { profesionales, clase media-media/media-alta. Sigan diariamente programas informativos en } \\
\text { televisión (TVE1, Antena 3, Tele 5). Madrid }\end{array}$ \\
\hline $\begin{array}{l}\text { Grupo de } \\
\text { Discusión } 2\end{array}$ & $\begin{array}{l}\text { Grupo de hombres y mujeres, ocupados, empleados del sector servicios y administrativos } \\
\text { (administrativos de banca o funcionarios), 50-60 años. Sigan diariamente informativos en } \\
\text { televisión (TVE1, Telemadrid, La 2). Madrid }\end{array}$ \\
\hline $\begin{array}{l}\text { Grupo de } \\
\text { Discusión } 3\end{array}$ & $\begin{array}{l}\text { Grupo de varones. La mitad trabajadores del sector industrial (metal, químico, etc.); la mitad } \\
\text { obreros autónomos (fontaneros, carpinteros, etc.), 25-35 años. Sigan diariamente informativos en } \\
\text { televisión. Madrid. }\end{array}$ \\
\hline $\begin{array}{l}\text { Grupo de } \\
\text { Discusión } 4\end{array}$ & $\begin{array}{l}\text { Grupo de mujeres, amas de casa. Clase media-media/media-baja. } 45-55 \text { años. Sigan diariamente } \\
\text { programas informativos en televisión (Cuatro, La Sexta, TVE.1). Madrid. }\end{array}$ \\
\hline $\begin{array}{l}\text { Grupo de } \\
\text { Discusión } 5\end{array}$ & $\begin{array}{l}\text { Grupo de jóvenes universitarios. Clase media-media. 19-24 años. La mitad estudiantes de } \\
\text { ciencias, la mitad estudiantes de letras o sociales (excepción estudiantes de Sociólogía o } \\
\text { cualquiera de las titulaciones relacionadas con Comunicación). Sigan diariamente informativos } \\
\text { en televisión. Madrid. }\end{array}$ \\
\hline $\begin{array}{l}\text { Grupo de } \\
\text { Discusión } 6\end{array}$ & $\begin{array}{l}\text { Grupo de hombres y mujeres, ocupados, entre } 40 \text { y } 50 \text { años, trabajen como profesionales, clase } \\
\text { media-alta. Sigan diariamente programas informativos en televisión (TVE1, Telemadrid, Antena } \\
\text { 3). Madrid }\end{array}$ \\
\hline
\end{tabular}

La dinámica estuvo estructurada alrededor de los programas de información que se seguían, razones para tal comportamiento y criterios tomados en la decisión, así como la percepción de cambios en este género televisivo. Así, la dinámica de las reuniones se ha estructurado a partir del siguiente guión básico:

- Introducción: prácticas en relación a los informativos de televisión.

- Opiniones generales sobre los principales informativos diarios.

- Diferencias apreciadas entre unos programas informativos y otros.

- Percepción de la evolución de los informativos en televisión. Principales cambios.

- Expectativas de futuro de los informativos en televisión.

Sobre este guión básico, hay que introducir las concreciones de todos estos apartados con respecto a uno de los objetivos específicos y centrales de la investigación, como la percepción de los cambios en los informativos de TVE a partir de su nuevo estatuto o el peso de la oposición entre cadenas privadas y públicas en la valoración de los programas informativos. No obstante, hay que subrayar la aplicación de esta guía, destinada más a constituirse en una serie de enganches temáticos dentro del discurso espontáneo de los grupos -como prescriben varios autores (Ibáñez, 1979; Ca- 
1lejo, 2000; Gutiérrez, 2008), que como una sucesión de preguntas. De aquí que las dinámicas fueran abiertas, especialmente en su fase inicial, destinada a hablar de la relación de los participantes con los programas informativos televisivos. Una primera fase que es la que ha generado el grueso del material discursivo que aquí ha servido para el análisis.

\section{Noticias de verdad como núcleo del informativo}

Desde el punto de vista de los temas, hay que destacar las discusiones centradas en la definición e identidad de noticia, derivadas de la generalizada consideración de que existen contenidos de los programas informativos televisivos que, aunque son presentados como noticia, genera dudas sobre tal identidad. Estos otros contenidos son referidos de distintas formas para señalar precisamente su distancia de lo que se considera noticias de verdad. En el siguiente fragmento discursivo, se denominan como rollo y se subraya como característica especial -negativa- de las mismas el hecho de que ya no responden al interés general sino a intereses más específicos o especializados: para ciertas personas...

- A mí en concreto lo que te mueve un poco es empieza el primer bloque de noticias, que digamos un poco lo que llama, no lo quito ahora sino [...] las cuatro o cinco primeras noticias y a raíz que pasa ahí luego ya viene el rollo, hay una exposición de arquitectura, tal, cual, que yo no digo que no sea interesante, pero a lo mejor ahora será interesante para ciertas personas que quieran concretamente eso, entonces... (Grupo de discusión 3)

Las no-noticias o menos-noticias, en la medida que no son consideradas correspondiendo al interés general, son recibidas desde la desconfianza. La percepción de una selección de contenidos informativos no regidos por el interés general conduce a preguntarse por la selección de los intereses seguidos y, por lo tanto, a un paso desde lo que se considera transparente -el interés general- a lo que aparece como opaco y, por lo tanto, base de la desconfianza. Si no corresponden al interés general, se proyecta que se corresponden con intereses particulares, ya sea de carácter político -ocultar lo importante- ya sea de carácter económico, articulándose a través del término vender, aquí entendido más como venta de audiencia que como seguimiento específico de intereses particulares de terceros - presentar lo que otros quieren vender, como publicidad oculta- aun cuando como puede observarse en el siguiente fragmento discursivo, la frontera que separa unos y otros puede ser débil.

- Sí, sí, un folletín porque al final cuando estás viendo las noticias, a los diez minutos de las noticias te están contando cosas que estás diciendo y esto es un telediario. Y nadie lo dice, porque es verdad que estás viendo y terminas diciendo, un telediario y estás viendo que si como cocinaba un plato, que si ahora el menú de la crisis, ahora el menú del restaurante de no sé qué y a mí precisamente para un informativo pues no pega, que vende eso..., debe de vender porque sino no lo echarían. (Grupo de discusión 3)

Una desconfianza que genera un círculo de desconfianza que se proyecta sobre el conjunto del informativo. Las no-noticia se perciben como táctica del programa para limitar la extensión de lo que son consideradas como noticias de verdad, atribuyén- 
dose una especie de táctica de calamar: para ocultar lo importante; o como táctica de relleno. Así se desarrolla en los siguientes fragmentos que, procediendo de distintos grupos de discusión, utilizan el mismo término para señalar a estas no-noticias: paja.

- Hombre, suelen meter mucha paja porque hay unas noticias tontísimas que están tres horas explicándolas y luego no te explican bien las cosas importantes. O sea las cosas que no quieren darles importancia no les dan importancia y para ellos... que en un comedor de Valencia haya habido un filete en mal estado están tres horas y entrevistan niño por niño y luego no te explican bien las cosas que te tienen que explicar bien (Grupo de discusión 5)

- Y hay que meter mucha paja, todos los días hay que hacer el informativo y te dura, no sé, cuánto, 45 minutos y hay que sacar noticias de las que son del día a día más luego... porque eso ahora a lo mejor se rellena tanto, te meten ahí un reportaje, pues ha habido unos trillizos en yo qué sé en Estados Unidos o en cualquier sitio o en el culo del mundo, pues te lo ponen, que eso no es una noticia que realmente a mí me importe mucho, pero hay que ir rellenando un poco (Grupo de discusión 3)

Es relevante que la discusión se centre en lo que debe ser considerado noticia y, por lo tanto, debe formar parte de un programa informativo diario, en una estrategia discursiva de los grupos por separar el grano de la paja, antes de entrar en la valoración de los formatos de noticia. Siendo la noticia caracterizada por el producto de una selección de lo importante de la realidad, en la medida que es lo importante para todos y sigue el interés general, se expulsa la no-noticia. A partir de aquí, la presencia de lo no importante traza sombras de sospecha y desconfianza sobre el conjunto de las unidades superiores a la noticia: programas o cadenas, como se observa en el siguiente fragmento discursivo.

- Yo por ejemplo Antena 3 no me gusta porque... aunque Matías Prats es bueno pero... bueno, me llamo Nuria, perdón, y no me gusta porque a veces parece del Tomate al final en el telediario y Matías Prats un poco se viene arriba cuando salen noticias en plan pues de modelos, de cosas de moda y sale bastante y creo que no es demasiado serio esa parte (Grupo de discusión 1)

Esta desconfianza a partir de la no-noticia impregna débilmente de desconfianza el resto de los contenidos del programa informativo, los que sí son percibidos como noticia. Puede hablarse de cierta impregnación salvada parcialmente, en la percepción de los públicos, por una especie de cortafuegos o dispositivo de reducción de la extensión de tal desconfianza derivado de la representación del programa informativo televisivo como una sucesión de bloques, de partes.

- Y tienes razón, yo creo que ahora lo que es el telediario en sí más que un telediario de dar unas noticias que parece que está dividido en dos partes, bueno, en tres partes, porque parece que es más importante lo de deportes que el resto de la información. Pero sí que entre medias de esta información, vamos yo me llama la atención que se habla de cocina, de platos de no sé qué; que bueno, que para ser un telediario no creo que sea el sitio más apropiado, creo que luego hay programas que pueden usar y tal, pero claro, lo poco que hay es programas de corazón nada más (Grupo de discusión 3) 


\section{La verdad de las noticias: elementos para la confianza en las noticias}

Separado, como se decía antes, el grano de la paja, se entra en las referencias a través de las cuales el público tiene como confiable una noticia. La relación con la realidad, como valor principal de la noticia, puede considerarse la fuente de todas sus valoraciones, incluyendo la proyección de confianza: "Que te den la noticia lo más exacta posible, más a la realidad" (Grupo de discusión 4). Puede decirse que este es el valor supremo de la noticia. La relación con la realidad puesto que es la función del sistema de comunicación organizadamente mediada. La contrastación de la noticia como núcleo de la mediación. De alguna forma, certificar la realidad. Especialmente en el caso de la televisión (Callejo, 1995). Por lo tanto, se confía en que los medios cuenten lo que pasa en el mundo cuando dan una noticia.

Hay que subrayar que la relación con la realidad, que puede extenderse hasta el grado de relación con la verdad, del ejercicio de los medios, no es puesta en duda por los participantes en las reuniones. A lo sumo, es cuestionada esa especie de segunda elaboración que se hace con relación a esa realidad.

- Pero no te cuentan nada raro, ¿no?, te cuentan la realidad.

- Sí, pero que yo creo que se ceban mucho en la catástrofe (Grupo de discusión 2)

Como se verá más adelante, se juzga con desigual criterio -en relación con la confianza- cuando esa elaboración secundaria es atribuida al medio concreto, a la institución mediática, o a los presentadores, situándose como dos polos distintos en la generación de confianza.

En la elaboración primaria de la noticia, se juzga por los participantes en las reuniones: a) que la noticia se refiere a algo ocurrido; b) relacionado con la selección de lo noticiable, que lo que se ha considerado noticia es suficientemente relevante, de interés público. Desde este punto de vista, es como si la noticia se impusiera al medio de comunicación. Más que de una selección de la realidad, se vive como una imposición del acontecimiento, sin existir apenas críticas, salvo en relación con la valoración de ciertas colas de los informativos, dedicadas a los comentarios de sociedad, como se ha señalado en el apartado anterior.

En la elaboración secundaria, relativa al sentido que se da a ese acontecimiento noticioso, ya sea por la institución -elaboración criticada por los grupos de discusiónya sea por los presentadores, se admite el alejamiento del valor verdad: "nada es verdad ni nada es mentira".

La elaboración secundaria se establece como una graduación sobre la elaboración primaria de la noticia. Una graduación que puede desarrollarse según el código "realidad dura" versus "realidad suave": "Pero la noticia se puede dar más negra o como es en realidad y Antena 3 por ejemplo la da más suave" (Grupo de discusión 4). En otro fragmento de ese mismo grupo, se hablará de crudeza de la realidad. Es decir, se trata de una elaboración secundaria no solo admitida sino demandada y que, por lo tanto, se confía que el medio de comunicación concreto -la cadena televisiva-realice. Una elaboración secundaria que no se percibe como alejamiento de la verdad. Entienden que la propia lógica del sistema de comunicación mediada, en un contexto de pluralidad y competencia entre medias, lleva a confiar en el mismo, como si ningún 
actor de tal sistema pudiera saltarse la verdad de la noticia sin ser puesto en evidencia.

- Además con tanta competencia es absurdo que mientan porque uno miente y el otro se va a echar encima. Lo hemos tenido hace poco con el caso este de Wyoming, o sea que van a ir a por él, o va a por a por él aquel informativo que no mienta. Yo creo que en general la gente... yo por lo menos... (Grupo de discusión 6)

Teniendo en cuenta el contexto por el que se confía de manera general en la noticia que forma parte de un sistema de comunicación mediada organizada plural, hay elementos que refuerzan tal confianza. El formato de noticia bajo la retórica del "estar ahí", concretado en la imagen de los corresponsales o enviados especiales en escenarios de acontecimientos fuera de los estudios genera mayor confianza. Se establece una directa relación entre lo que ven -los profesionales que están ahí- y el mensaje -noticia- que narra lo visto.

- Porque realmente son los que te informan de o que está pasando en un sitio lejano

- De primera mano

- Da credibilidad

- Y yo creo que son más neutrales los enviados porque te dicen lo que ven (Grupo de discusión 5)

La piedra angular de la fuente de confianza en el caso de la noticia en televisión son los presentadores. La elaboración secundaria producida por los presentadores o conductores de los programas informativos se convierte en la principal fuente de confianza. Ello hace que, en función de la percepción de los presentadores, pudiera establecerse la relación con los informativos con mayor o menor confianza.

- Y se agradece mucho la espontaneidad por parte de los presentadores en determinado momento, tanto guión, tanto leer, termina siendo un poco aburrido. Por eso yo creo que a mí Matías Prats en concreto me gusta porque de repente suelta ahí cualquier tontería, pero por lo menos dices menos mal, la espontaneidad. La espontaneidad también... que limitarse tanto a un guión (Grupo de discusión 1)

Formalmente, hay una confianza general en que lo que se cuenta que ha sucedido, derivada del cruce de eco entre los distintos informativos: un todos hablan de la misma $\cos a$. Como si todos realizaran la misma elaboración primaria. Se trataría de la confianza en el sistema a partir de la convergencia en referencias. Sin embargo, la confianza con un informativo en particular aparece muy condicionada por la mediación de los presentadores o conductores de los programas.

- La diversidad. Es distinto pues desde como te están enfocando la noticia, la sensación de que realmente me están informando de algo que ha pasado y ya está, que eso también que no es verdad, porque en el informativo de Mara Torres suelen ser bastante críticos y meten su opinión y todo, pero bueno (Grupo de discusión 1)

Por lo tanto, la confianza reposa tanto en la objetividad del eco entrecruzado con respecto a la selección de los acontecimientos, y por la subjetividad-invertida por el men- 
saje, recibida por el receptor-mediadora de los presentadores. Una mediación en la que la opinión -de los presentadores- asumida como opinión de ellos -y no de la institución mediática en la que desarrollan su labor- se convierte en fuente de confianza.

El comentario subjetivo o incorporación de subjetividad -a través del cuerpo del presentador- a la noticia, se convierte en un valor de confianza para la noticia. Se comenta lo que es verdad, sobre lo que es verdad. Es decir, realizando una elaboración secundaria (opinión, comentario) sobre la noticia, la verdad de ésta o estado primario de la misma (su selección y su relación con la realidad) quedan reafirmadas.

Por lo tanto, la (falta) de importancia, como fuente de desconfianza, en la medida que oculta otros temas o, al menos, les da el mismo grado de importancia, sin jerarquizar.

- Yo creo que cuando hay un problema dan más importancia a otros temas, cuando hay un problema importante en el país, como que le [sic] aparcan y dan de otro tema (Grupo de discusión 4)

O una desconfianza surgida de la atribución de intereses particulares-económicos a la institución mediática, que la llevarían a ponerlos por encima del interés público.

- ...para mi un telediario en Prime Time pues no me parece nada interesante porque creo que están un poco supeditados a la publicidad y a los picos de audiencia y a todo esto y entonces eso claramente ahí ya te está marcando que tienes que dar una noticia (Grupo de discusión 1)

\section{Conclusiones}

Puede hablarse de un doble contrato de confianza o doble nivel del contrato de confianza entre noticias televisivas y sociedad, concretada como audiencia televisiva. Por un lado, el derivado de la elaboración primaria de la noticia y que hay que entroncar con la confianza en el sistema de comunicación mediada organizada. Desde este punto de vista, la desconfianza en el sistema puede considerarse de grado menor, lo que hace que cuando se pregunta de forma generalizada -sobre todos los medios de comunicación- tal y como se hace en las encuestas con cuestionario estandarizado, el grado de confianza en los medios sea comparativamente alto. Las declaraciones de desconfianza surgidas parecen derivar de la interrupción del seguimiento a los acontecimientos. Se percibe cierta contradicción entre la selección de un acontecimiento como relevante -como suficientemente relevante como para acceder a los informativos televisivos- pudiendo estar en la portada durante varios días; hasta que, casi de manera repentina, se interrumpe el flujo de información sobre tal acontecimiento, se deja de seguir. Se proyecta así una percepción de cierta arbitrariedad en lo que hacen los medios. En primer lugar, como creadores de un acontecimiento, lo que hacen es darle durabilidad. Como plantean Moloch y Lester (1997) un acontecimiento es el producto de la durabilidad de su presencia en el sistema de medios de comunicación.

No puede hablarse de confianza ciega en un medio, ni siquiera en el conjunto del sistema de comunicación mediada. Tal vez el concepto más conveniente es el de confianza crítica, ya apuntado por Simmel (2013) hace un siglo y retomado por quienes plantean que estamos en sociedades de la post-confianza 
- Pero es muy peligroso porque realmente vas guiado, o sea has visto esa media hora y tu información de ese día del mundo es esa media hora de noticias y es muy peligroso, desgraciadamente muchas veces es así. Pero sobre todo es para estar informado, para ver un poco lo que pasa en el mundo (Grupo de discusión 1)

Por otro lado, un contrato de confianza con los profesiones de los medios de comunicación, ya sean estos profesionales los presentadores del programa o los informadores desde los escenarios de la noticia, enviados o reporteros. Con los que realizan la elaboración secundaria de la noticia.

- O sea a mí me gustan los presentadores, sobre todo, la manera de comunicar, porque las noticias son las mismas (Grupo de discusión 2)

El sistema de comunicación mediada institucionalizada produce la elaboración primaria concretada en la selección de la realidad por la noticia: es lo que ha ocurrido porque todos los medios de comunicación del sistema presentan la misma noticia. La elaboración secundaria y el refuerzo-reducción de la confianza de la noticia deriva de los profesionales y las instituciones mediadoras: dirección de programas, cadena, etc. Desde tal división, los profesionales actúan como vías de acceso (Giddens, 1990) al sistema de confianza y como concreciones directas de tal sistema. Es decir, son los que administran ante los usuarios (telespectadores) la confianza puesta en el sistema y, a la vez, quienes inyectan confianza en el sistema. Por ello y como aparece en el siguiente fragmento discursivo, su elaboración secundaria en la actualidad se ve enmarcada en un relativamente alto grado de confianza, lo que incluye la realización de comentarios que pueden considerarse personales:

- Yo no creo que no se sea objetivo porque el periodista dé la noticia desde su punto de vista, yo creo que donde se manipula la información no es en el periodista, yo creo que es por encima de él, o sea, la cadena, el director, pues ahí sería donde habría que actuar, porque tú puedes ser periodista y contarlo con unas palabras o con otras, pero si eres neutral y tal, no tiene porqué verse la noticia... (Grupo de discusión 5)

Otra cosa distinta, como aparece en el fragmento anterior, es la elaboración secundaria realizada por el programa -alusión a la dirección del programa-o la cadena. Es aquí donde se genera la desconfianza. ¿Cómo puede articularse la confianza en los profesionales y el sistema y la relativa desconfianza en las instituciones mediadoras, como el programa o la cadena, cuando se sitúa a éstas por encima de los profesionales? Para interpretar esta situación y dar respuesta a esta pregunta, hay que retornar a la práctica de la selección de los contenidos de los informativos televisivos y lo que se considera noticia y no-noticia. Se confía en lo que es admitido como noticia, lo que se considera que da cuenta de una realidad importante y, por tanto, del interés general porque está en el sistema de comunicación mediada institucionalizada en la medida que todos los medios de comunicación la dan. La pueden dar de distinta forma en función de sus profesionales, sin que ello reduzca la confianza sobre la noticia o el medio, sino, al contrario, la confianza en los profesionales amplia la confianza sobre la noticia. Es la presencia de no-noticia, de contenidos de "paja", lo que genera un círculo 
de desconfianza que, a su vez, puede verse alimentado por la desconfianza en instituciones como la cadena.

Más acá de la confianza o desconfianza con respecto a un específico medio de comunicación, se confía en el sistema de medios de comunicación institucionalizada, en la propia pluralidad, en la idea de que todo lo que diga un medio de comunicación está fiscalizado por estos medios, por el sistema. La pluralidad, lo que incluye la división con respecto a un acontecimiento de los distintos actores presentes en el sistema de comunicación mediada institucionalizada, no solo no disminuye la confianza en el mismo sino que la aumenta, pues, aunque algún medio concreto pudiera quedar desacreditado, es como si el conjunto del sistema, al actuar compactamente se corrigiera a sí mismo agotando esa realidad, hasta completarla totalmente. Agotan el tema, que es lo definitivo y no las opiniones (Luhmann, 2000: 101).

¿Cabría otra posibilidad? ¿Cabría la desconfianza en el sistema de medios de comunicación institucionalizada? Difícilmente. Como se dice en el siguiente fragmento discursivo, es la única forma de enterarte

- A mí sí me interesan, me parece que al margen de que estén vendidos o no estén ven-

didos es la única forma de enterarte de lo que está pasando en cualquier parte del mundo

(Grupo de discusión 5)

Al menos que pudiéramos vivir sin información sobre lo que pasa en el mundo, con lo que significaría de indiferencia sobre el mundo. ¿Puede ocurrir esto en las sociedades avanzadas? El daño resultante de una ruptura de la confianza con el sistema de comunicación mediada institucionalmente puede ser más grande que el beneficio que se gana por el hecho de no creer en lo que dicen. Si no se confiara en el sistema de comunicación mediada institucionalmente habría que ir a comprobar cada noticia. Es el propio sistema de comunicación mediada institucionalmente el que, con la repetición de las mismas referencias - la "misma noticia"- por parte de distintos medios de comunicación el que genera la confianza en sí mismo. Los medios de comunicación han de configurase como sistema que dialoga entre sí: "cuando los medios masivos incurren en el monólogo, provocan desconfianza antes que confianza, y se encuentran otros medios para destruir su monopolio" (McBride, 1993: 45).

La confianza en el sistema de medios de comunicación institucionalizada puede entenderse como confianza orgánica. Confianza en que este sistema social cumple con su función.

Una última reflexión vinculada con la lógica de funcionamiento de nuestras sociedades avanzadas. Los medios de comunicación, que son los que en mayor medida generan desconfianza e incertidumbre de una manera generalizada, sobre el conjunto de nuestras realidades circundantes hasta alcanzarse un estado de vulnerabilidad de la confianza (Silverstone, 2007: 42), producen, como una parte de su propia forma de funcionamiento, confianza sobre ellos mismos, en cuanto elementos del sistema de comunicación mediada institucionalmente. Y lo hacen, como hemos visto, principalmente a partir de la elaboración secundaria que llevan a cabo los profesionales de lo que se considera la noticia por los públicos. Esta confianza general (sistema) y concreta (profesionales que aparecen en la imagen televisiva, firmando la información) tiene sus agu- 
jeros negros y, por lo tanto, sus posibles fuentes de desconfianza, como ocurre cuando en los programas informativos se incluyen contenidos que se consideran que responden más a intereses particulares (políticos o comerciales) que al interés general, poniéndose el acento en la percepción de desconfianza en la selección de tales contenidos. Es decir, el contrato de confianza de los públicos no lo realizan tanto con el medio de comunicación concreto, con la cadena de televisión, como pretende Nourgayréde, como con el conjunto del sistema y los profesionales que dan la cara a la noticia.

\section{Referencias bibliográficas}

ALMOND, Gabriel y VERBA, Sidney (1989): The Civic Culture: Political Attitudes and Democracy in Five Nations. London, Sage (e.o. 1963).

BENITO, Ángel (1995): La invención de la actualidad. Madrid, Fondo Cultura Económica.

BLUMLER, Jay G. (ed., 1993): Televisión e interés público. Barcelona, Bosch.

BOURDIEU, Pierre (1988): La distinction. Madrid, Taurus.

BRANTS, Kees (1998): "Who's Afraid of Infotainment? European Journal of Communication, 13 (3), pp. 315-335.

FRANKLIN, Bob (1997): Newszak and news media. London, Arnold.

LANGER, John (1998): La televisión sensacionalista. El periodismo popular y las "otras noticias". Barcelona, Paidós.

BRIGGS, Asa y BURKE, Peter (2002): De Gutenberg a Internet. Madrid, Taurus.

CALLEJO, Javier (2000): Introducción a una práctica de investigación. Barcelona, Paidós.

CLAUSSE, Roger (1961): Bosquejo de una sociología de la noticia. Caracas, Universidad Central de Venezuela.

FRANK, Joseph (1961): The Beginnings of the English Newspaper, 1620-1660. Cambridge, Harvard University Press.

GALTUNG, Johan y RUGE Mari Holmboe (1965): "The Structure of Foreign News". Journal of Peace Research 2 (1), pp. 64-90.

GIDDENS, Anthony (1990): The consequences of modernity. Cambridge, Polity Press.

GUTIÉRREZ, Juan (2008): Dinámica del grupo de discusión. Madrid, CIS.

IBÁÑEZ, Jesús (1979): Más allá de la sociología. Madrid, Siglo XXI.

INGLEHART, Ronald (1999): "Trust, well-being and democracy", en WARREN, Mark E. (ed.): Democracy and Trust. Cambridge, Cambridge University Press, pp. 88-120.

LAHIRE, Bernard (2006): La culture des individus: dissonances culturelles et distinction de soi. Rennes, Presses Universitaires de Rennes. 
LASSWELL, Harold (1982): "Estructura y función de la comunicación en la sociedad", en MORAGAS, Miquel de (ed.): Sociología de la comunicación de masas. Barcelona: Gustavo Gili, pp. 192-206 (e.o. 1955).

LIVINGSTONE, Sonia (2002): Young People and New Media. London, Sage.

LLEDÓ, Emilio (1998): El silencio de la escritura. Madrid, Espasa Calpe.

LÖFSTEDT, Ragnar E. (2005): Risk Magement in Post-Trust Societies. London, Earthscan.

LUHMANN, Niklas (1996): Confianza. Barcelona, Anthropos.

LUHMANN, Niklas (2000): La realidad de los medios de masas. Barcelona, Anthropos.

MAIGRET, Eric (2005): Sociología de la comunicación y de los medios. México, Fondo de Cultura Económica.

MCBRIDE, Sean (1993): Un solo mundo, voces múltiples. México, Fondo de Cultura Económica.

MCMANUS, John H. (1994): Market-driven journalism: Let the citizen beware. London, Sage.

MCQUAIL, Dennis (1991): Introducción a la teoría de la comunicación de masas. Barcelona, Paidós.

MOLOTCH, Harvey \& LESTER, Marilyn (1997): "News as purposive behaviour: On the strategic use of routine events, accidents and scandals", en BERKOWITZ, Daniel (ed.): Social meaning of news. Sage Publications, Thousand Oaks, CA, pp. 193-209.

MOTT, Frank L. (1962): American Journalism: A history, 1690-1960. New York, McMillan.

PUTNAM, Robert David (1995): “Tuning In, Tuning Out: The Strange Dissapearance of Social Capital in America". Political Science and Politics, vol. 28 (4): pp. 664-683.

SCHUDSON, Michael (1978): Discovering the News: A Social History of American Newspaper. New York, Basic Books.

SILVERSTONE, Roger (2007): Media and Morality. Cambridge, Polity.

SIMMEL, George (2013): Filosofía del dinero. Madrid, Capitán Swing.

STZOMPKA, Piotr (1999): Trust: A Sociological Theory. Cambridge, Cambridge University Press.

THUDEROZ, Christian (2003): "Introduction au propos: la confiance en question", en MANGEMATIN, Vincent y THUDEROZ, Christian (dir.): Des mondes de confiance. Paris, CNRS, pp. 19-30.

THUSSU, Daya Kishan (2007): News as Entertainment. London, Sage.

TUCHMAN, Gaye (1983): La producción de la noticia. Estudio sobre la construcción social de la realidad. Barcelona, Gustavo Gili. 
WALLS, John; PIDGEON, Nick; WEYMAN, Andrew et al. (2004): "Critical trust: understanding lay perceptions of health and safety risk regulation". Health, Risk \& Society, 6 (2), pp.133-150.

WINSTON, Brian (2002): “Towards Tabloidization? Glasgow Revisited, 1975-2001". Journalism Studies, 3 (1): 5-20.

WOLF, Mauro (1987): La investigación de comunicación de masas, Barcelona, Paidós.

ZIMAN, John (2000): "Evolutionary models of technological change", en ZIMAN, John (ed.): Technological Innovation as an Evolutionary Process. Cambridge, The Press Syndicate of the University of Cambridge, pp. 3-12. 